\title{
MASS TRANSFER THROUGH RAREFIED GAS BETWEEN CONCENTRIC SPHERES
}

RYOZO TOEI, MORIO OKAZAKI, AKIRA URAGAMI AND YUJI TAKAKI

Department of Chemical Engineering, Kyoto University, Kyoto

\begin{abstract}
A theoretical analysis of mass transfer under a reduced pressure, especially the transition regime was made on the basis of the gas kinetic theory. Maxwell moment method utilizing the two-sided Maxwellian distribution function proposed by Lees et al. was applied to the mass transfer between two concentric spheres, and the accomodation cosficient was taken into account. The analytical solution with the evaluated accomodation coafficient 0.9 agread well with the experimental results of sublimation of naphthalene performed by the authors.
\end{abstract}

\section{Introduction}

Mass transfer operations under reduced pressures, such as freeze-drying, molecular distillation, metallizing under vacuum and so on, are accompanied with phase change in all cases and play a very important roll in chemical engineering. Considering the mass transfer phenomena with phase change, for example, sublimation, condensation and evaporation, two kinds of resistance for mass transfer should be evaluated. One is the resistance through the surrounding space, so called the resistance of gaseous diffusion and the other is the one for phase change at the interface, which is concerned in accomodation, condensation or evaporation coefficients.

For mass transfer through the surrounding space three regimes classified by the Knudsen number, $K n$, have been considered. Those are the free molecular regime under highly rarefied condition, $K n>10$, the continuum regime under ordinary condition, $K n<0.01$, and the transition regime between them, $0.01<K n<10$. The mass transfer under this transition regime has given a great interest to investigators of physics and technology. Especially its applications are very important for chemical engineering but few theoretical and experimental works ${ }^{3,4,8,11)}$ have been performed because of its complexity.

It has been generally accepted apriori by the previous investigators $^{8)}$ that Stefan's equation with the pressurejump boundary condition which is analogous to MaxwellSmoluchowski's temperature-jump boundary condition for heat transfer would be correct. But there has been no theoretical basis on applying Stefan's equation to the mass transfer with large degree of rarefaction, high mass flux and large pressure gradient between evaporating (or sublimating) and condensating surface.

The present work was undertaken to derive a theoretical equation of mass transfer under reduced pressures, especially transition regime, through the gas kinetic theory and compare with the experimental results.

The Maxwell moment method utilizing the two-sided Maxwellian distribution function ${ }^{2,6,7)}$ was applied to the mass transfer between concentric spheres. The analytical

\footnotetext{
* Received on November 30, 1967
}

solution obtained was compared with the experimental results obtained by the sublimation of naphthalene under reduced pressures $\left(10^{-1} \sim 10^{-3} \mathrm{mmHg}\right.$ in total pressure).

\section{Theoretical Works}

1-1. Distribution Function and Mean Quantities

Let us consider a sphere of radius $R_{I}$ placed at the center of a hollow sphere of radius $R_{\mathbb{I}}$, with $R_{\mathrm{I}}<R_{\mathbb{I}}$ (Fig. 1).

Sublimation (or evaporation) of molecule $A$ occurs at the outer surface of the inner sphere and condensation at the inner surface of the outer sphere. The annular region $\left(R_{\mathrm{I}}<R<R_{\mathbb{I I}}\right)$ is filled with diffusion gas $A$ and innert gas $B$.

By applying Lee's model ${ }^{2)}$, the "two-sided Maxwellian" velocity distribution functions are defined at an arbitrary point in the annular space as follows (Fig. 1); all outwardly directed molecules with velocity vector $\boldsymbol{\xi}$,

$$
\begin{gathered}
\xi=\left(\xi_{R}{ }^{2}+\xi_{\theta}{ }^{2}+\xi_{\phi}{ }^{2}\right)^{1 / 2} \\
\phi=\tan ^{-1}\left\{\left(\xi_{\theta}{ }^{2}+\xi_{\phi}{ }^{2}\right)^{1 / 2} \xi_{R}{ }^{-1}\right\}
\end{gathered}
$$

lying inside "the wedge of influence" (region 1 in Fig.

1) are characterized by one Maxwellian $f_{1}$,

$$
f=f_{1}=n_{1}\left(\frac{m}{2 \pi k T}\right)^{3 / 2} \exp \left(-\frac{m \xi^{2}}{2 k T}\right)
$$

for

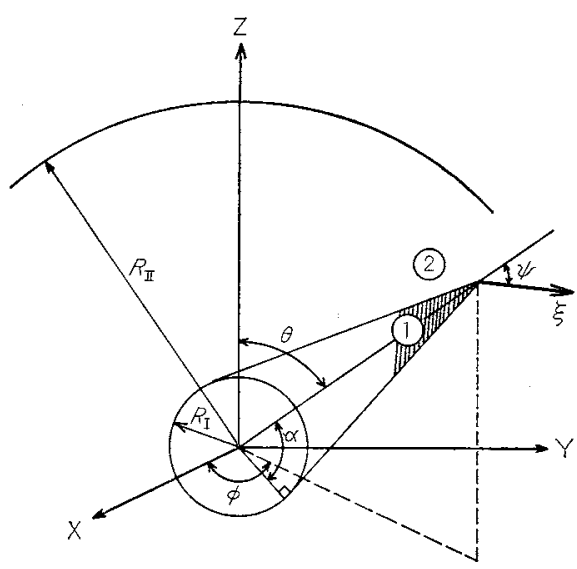

Fig. I Spherical polar coordinates 


$$
-\frac{\pi}{2}+\alpha<\phi<\frac{\pi}{2}-\alpha
$$

in which

$$
\alpha=\cos ^{-1}\left(\frac{R_{\mathbf{I}}}{R}\right)
$$

Then, all molecules with velocity vector $\boldsymbol{\xi}$ lying outside of region 1 are characterized by $f_{2}$, i. e.,

$$
f=f_{2}=n_{2}\left(\frac{m}{2 \pi \overline{k T}}\right)^{3 / 2} \exp \left(-\frac{m \xi^{2}}{2 k \bar{T}}\right)
$$

for

$$
\frac{\pi}{2}-\alpha<\phi<\frac{3}{2} \pi-\alpha
$$

where $n_{1}(R)$ and $n_{2}(R)$ are unknown functions of radial distance.

In the above definitions, we consider the case that the temperature difference between two spheres is small so the each temperature of two spheres can be approximately denoted as the same absolute temperature $T\left[{ }^{\circ} \mathrm{K}\right]$. The Maxwellian velocity distribution for stationary gas is assumed applicable when the net flow of molecule $A$ between concentric spheres is relatively small.

Knowing the distribution function $f$, one can evaluate all mean quantities $\langle Q\rangle$ by averaging over whole velocity space,

$$
\begin{aligned}
<Q> & =\int Q f d \boldsymbol{\xi}=\int_{\mathbf{I}} Q f_{1} d \boldsymbol{\xi}+\int_{\mathbf{I}} Q f_{2} d \boldsymbol{\xi} \\
\int f d \boldsymbol{\xi}=n & =\frac{1}{2}\left\{n_{1}(1-\sin \alpha)+n_{2}(1+\sin \alpha)\right\} \\
\int f_{\xi_{R}} d \boldsymbol{\xi}=u_{R} & =\frac{1}{4}\left(\frac{2 k T}{m \pi}\right)^{1 / 2}(1+\cos 2 \alpha)\left(n_{1}-n_{2}\right) \\
-\int m \xi_{R}^{2} d \boldsymbol{\xi} & =P_{R R} \\
& =-\frac{k T}{2}\left\{n_{1}\left(1-\sin ^{3} \alpha\right)+n_{2}\left(1+\sin ^{3} \alpha\right)\right\} \\
p & =-\frac{1}{3}\left\{P_{R R}+P_{\theta \theta}+P_{\phi \phi}\right\} \\
& =\frac{k T}{2}\left\{n_{1}(1-\sin \alpha)+n_{2}(1+\sin \alpha)\right\}
\end{aligned}
$$

\section{1-2. The Maxwell Integral Equation of Transfer}

In spherical co-ordinates the Maxwell integral equation of transfer for the spherical symmetric case is as follows:

$$
\begin{aligned}
\frac{1}{R^{2}} & \frac{\partial}{\partial R}\left(R^{2} \int f \xi_{R} Q d \xi\right)+\frac{1}{R \tan \theta} \int f \xi_{\theta} Q d \xi \\
& -\int f\left(\frac{\xi_{0}^{2}}{R}+\frac{\xi_{\phi}^{2}}{R}\right) \frac{\partial Q}{\partial \xi_{R}} d \xi+\int f\left(\frac{\xi_{R} \xi_{\theta}}{R}\right. \\
& \left.-\frac{\xi_{\phi}^{2}}{R \tan \theta}\right) \frac{\partial Q}{\partial \xi_{\theta}} d \xi+\int f\left(\frac{\xi_{R} \xi_{\phi}}{R}+\frac{\xi_{\theta} \xi_{\phi}}{R \tan \theta}\right) \frac{\partial Q}{\partial \xi_{\phi}} d \xi \\
& =\Delta Q
\end{aligned}
$$

where $\Delta Q$ is the collision integral.

There are two kinds of molecule, diffusing molecule $A$ and non-diffusing molecule $B$ and one should take the distribution functions for each molecule into account. Therefore there are four unknown parameters, $n_{A 1}, n_{A 2}$, $n_{B 1}$ and $n_{B 2}$, and they could be evaluated by utilizing four Maxwell integral equations as follows.

(a) Mass Conservation for Molecule $A$ Setting $Q$ $=m_{A}$, we find $\Delta Q=0$ because the mass is invariant during collisions, and obtain the ordinary continuity equation as follows;

$$
\frac{1}{R^{2}} \frac{\partial}{\partial R}\left(R^{2} \int f_{A} m_{A} \xi_{A R} d \xi_{A}\right)=0
$$

From Eqs. (8) and (12), we obtain the following relation;

$$
\begin{aligned}
& \frac{1}{4 R^{2}}\left(\frac{2 m_{A} k}{\pi}\right)^{1 / 2} \frac{\partial}{\partial R}\left\{R^{2}(1+\cos 2 \alpha)\right. \\
& \left.\left(n_{A 1}-n_{A 2}\right) T^{1 / 2}\right\}=0
\end{aligned}
$$

On the other hand;

$$
R^{2}(1+\cos 2 \alpha)=2 R_{\mathbf{I}}^{2}=\text { const. }
$$

then the following equation is obtained,

$$
n_{A 1}-n_{A 2}=\gamma^{\prime}=\text { const. }
$$

where $\gamma^{\prime}$ is an integral constant.

(b) Mass Conservation for Molecule $B$

Setting $Q=m_{B}$,

$$
\begin{aligned}
& \frac{1}{4 R^{2}}\left(\frac{2 m_{B} k}{\pi}\right)^{1 / 2} \frac{\partial}{\partial R}\left\{R^{2}(1+\cos 2 \alpha)\right. \\
& \left.\left(n_{B 1}-n_{B 2}\right) T^{1 / 2}\right\}=0
\end{aligned}
$$

Here molecule $B$ is the non-diffusing component, then

$$
n_{B 1}-n_{B 2}=0
$$

(c) $R$-momentum Conservation Summing up the momentum transfer equations for molecule $A$ and $B$, the collision integral becomes zero as the $R$-momentum is conservative as a whole. Substituting $Q=m_{A} \xi_{A R}$ or $Q=$ $m_{B} \xi_{B R}$ into Eq. (11),

$$
\begin{aligned}
& \frac{\partial}{\partial R}\left(\int f_{A} m_{A} \xi_{A R}{ }^{2} d \xi\right)+\frac{3}{R} \int\left\{-\frac{1}{3}\left(f_{A} m_{A} \xi_{A R}{ }^{2}+f_{A} m_{A} \xi_{A \theta^{2}}{ }^{2}\right.\right. \\
& \left.\left.\quad+f_{A} m_{A} \xi_{A \phi}{ }^{2}\right)+f_{A} m_{A} \xi_{A R}{ }^{2}\right\} d \xi=\Delta Q_{A B}
\end{aligned}
$$

Then we obtain the next equation,

$$
\begin{aligned}
& \frac{d}{d R}\left\{\left(n_{A 1}+n_{A 2}\right) T\right\}-\sin ^{3} \alpha \frac{d}{d R}\left\{\left(n_{A 1}-n_{A 2}\right) T\right\} \\
& \quad=\frac{2}{k} \Delta Q_{A B}
\end{aligned}
$$

likewise

$$
\begin{aligned}
& \frac{d}{d R}\left\{\left(n_{B 1}+n_{B 2}\right) T\right\}-\sin ^{3} \alpha \frac{d}{d R} \cdot\left\{\left(n_{B 1}-n_{B 2}\right) T\right\} \\
& \quad=\frac{2}{k} \Delta Q_{B A}
\end{aligned}
$$

Summing up Eqs. (18) and (19),

$$
\begin{gathered}
d \\
d R\left(n_{A 1}+n_{A 2}+n_{B 1}+n_{B 2}\right)=0 \\
n_{A 1}+n_{A 2}+n_{B 1}+n_{B 2}=\delta^{\prime}=\text { const. }
\end{gathered}
$$

where $\delta^{\prime}$ is an integral constant.

(d) Mass Flux for Molecule $A^{5)}$ Since we are primarily interested in radial mass transfer, the mass flux equation is given by taking $Q=m_{A} \xi_{A R}$. In the mass flux equation, the collision integral $\Delta Q_{A B}$ is evaluated with Maxwell's inverse-fifth-power force law $F=\widetilde{K}_{A B} /$ $r^{55}$, for simplicity. Then it should be noted that mass flux is influenced only by the collision integral $\Delta Q_{A B}$ involving the collisions between molecule $A$ and $B$.

Assuming that a molecule $A$ collides with a molecule $B$ from a direction $P$ (see Fig. 2), the collision integral $\triangle Q_{A B}$ is expressed as follows:

$$
\Delta Q_{A B}=\iiint \int\left(Q^{\prime}-Q\right) f_{A} f_{B} V d \xi_{A} d \xi_{B} b d b d \varepsilon
$$

and by Maxwell ${ }^{5}$,

$$
\begin{aligned}
\int_{0}^{2 \pi}\left(Q^{\prime}-Q\right) d \varepsilon & =\int_{0}^{2 \pi} m_{A}\left(\xi_{A R}{ }^{\prime}-\xi_{A R}\right) d \varepsilon \\
& =\frac{m_{A} m_{B}}{m_{A}+m_{B}}\left(\xi_{B R}-\xi_{A R}\right) 4 \pi \sin ^{2} \theta^{\prime} \\
b d b & =\left\{\frac{\widetilde{K}_{A B}\left(m_{A}+m_{B}\right)}{V^{2} m_{A} m_{B}}\right\}^{1 / 2} \beta d \beta \\
A_{1} & =\int_{0}^{\infty} 4 \pi \beta d \beta \sin ^{2} \theta^{\prime}=2.6595
\end{aligned}
$$


Therefore

$$
\begin{aligned}
\Delta Q_{A B} & =A_{1}\left(\frac{m_{A} m_{B}}{m_{A}+m_{B}} \widetilde{K}_{A B}\right)^{1 / 2} \iint f_{A} f_{B}\left(\xi_{B R}-\xi_{A R}\right) d \xi_{A} d \xi_{B} \\
& =A_{1}\left(\frac{m_{A} m_{B}}{m_{A}+m_{B}} \widetilde{K}_{A B}\right)^{1 / 2}\left(n_{A} u_{B R}-n_{B} u_{A R}\right)
\end{aligned}
$$

Taking molecule $B$ being at rest into account :

$$
\begin{aligned}
\Delta Q_{A B} & =-A_{1}\left(\frac{m_{A} m_{B}}{m_{A}+m_{B}} \widetilde{K}_{A B}\right)^{1 / 2} u_{A R} n_{B} \\
& =-A_{1}\left(\frac{m_{B} k T \widetilde{K}_{A B}}{2 \pi\left(m_{A}+m_{B}\right)}\right\}^{1 / 2} \gamma^{\prime} n_{B}\left(\frac{R_{\mathbf{I}}}{R}\right)^{2}
\end{aligned}
$$

The diffusion coefficient for the Maxwell molecule is evaluated by the following equation ${ }^{1)}$,

$$
\left[D_{A B}\right]=\frac{3 \pi}{4 n A_{1} \Gamma\left(2 \frac{1}{2}\right)}\left\{\frac{k T\left(m_{A}+m_{B}\right)}{2 \pi m_{A} m_{B}}\right\}^{1 / 2}\left(\frac{2 k T}{\widetilde{K}_{A B}}\right)^{1 / 2}
$$

Eliminating $\widetilde{K}_{A B}$ of Eq. (27) using Eq. (28), we obtain

$$
\begin{gathered}
\Delta Q_{A B}=-\frac{k T n_{B 1} \gamma^{l}}{2 n\left[D_{A B}\right]}\left(\frac{2 k T}{m_{A \pi}}\right)^{1 / 2}\left(\frac{R_{\mathbf{I}}}{R}\right)^{2} \\
\frac{d}{d \bar{R}}\left(n_{A 1}+n_{A 2}\right)=-\frac{R_{\mathrm{I}}}{n\left[D_{A B}\right]}\left(\frac{2 k T}{m_{A} \pi}\right)^{1 / 2} n_{B 1} \gamma^{\prime}\left(-\frac{1}{\bar{R}}\right)^{2}
\end{gathered}
$$

where

$$
\bar{R}=\frac{R}{R_{1}}
$$

Converting $n$ to $p$ by utilizing the relation $p=n k T$, we obtain the following set of equations;

$$
\begin{gathered}
p_{A 1}-p_{A 2}=\gamma=\text { const. } \\
p_{B 1}-p_{B 2}=0 \\
p_{A 1}+p_{A 2}+p_{B 1}+p_{B 2}=\delta=\text { const. } \\
\frac{d}{d \bar{R}}\left(\frac{p_{A 1}+p_{A 2}}{2}\right)=-\zeta \gamma p_{B 1}\left(\frac{1}{\bar{R}}\right)^{2}
\end{gathered}
$$

where

$$
\zeta=\frac{R_{\mathrm{r}}}{2 p_{i}\left[D_{A B}\right]}\left(\frac{2 k T}{m_{A} \pi}\right)^{1 / 2}=\text { const. }
$$

Then the following equations are obtained from Eqs. (31) $\sim(34)$,

$$
\begin{aligned}
& p_{A 1}=\frac{1}{2}\left\{\gamma+\delta+M \exp \left(-\frac{\zeta \gamma}{\bar{R}}\right)\right\} \\
& p_{A 2}=\frac{1}{2}\left\{-\gamma+\delta+M \exp \left(-\frac{\zeta \gamma}{\bar{R}}\right)\right\}
\end{aligned}
$$

and

$$
p_{B 1}=p_{B 2}=-\frac{M}{2} \exp \left(-\frac{\zeta \gamma}{\bar{R}}\right)
$$

where $M$ is an integral constant.

The set of Eqs. (36) (38), can be solved by using the boundary conditions in 1-3.

1-3. Boundary Conditions and Solutions

On defining the accomodation coefficient for mass transfer, it was assumed that when a number of molecules are impinging on a surface, the fraction $a$ of them are stuck and condensated, and the fraction $(1-a)$ are reflected diffusely.

Then

$$
\begin{aligned}
& \text { at } \bar{R}=\frac{R_{\mathbf{I}}}{R_{\mathbf{I}}}=1 ; p_{A 1}=p_{A 1 \mathbf{I}}=(1-a) p_{A 2 \mathbf{I}}+a p_{A I} \\
& \text { at } \bar{R}=\frac{R_{\mathbb{I}}}{R_{\mathbf{I}}}=\bar{R}^{*} ; \\
& p_{A 2}=p_{A 2 \mathbf{I}}=\frac{1-a}{2}\left\{\left(1+\cos 2 \alpha_{\mathbb{I I}}\right) p_{A 1 \mathbf{I}}\right. \\
& \left.\quad+\left(1-\cos 2 \alpha_{\mathbb{I}}\right) p_{A 2 \mathbb{I}}\right\}+a p_{A \mathbf{I}}
\end{aligned}
$$

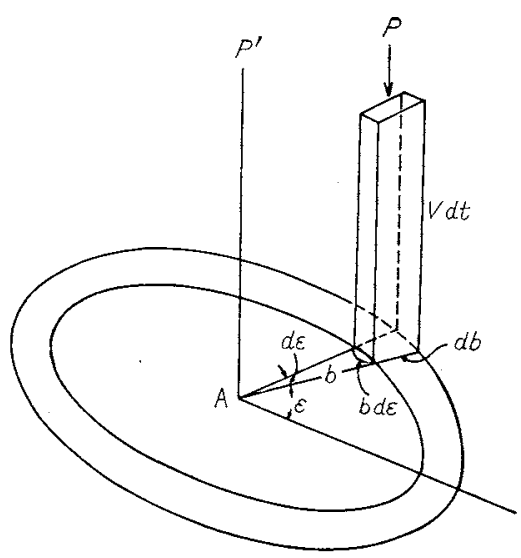

Fig. 2 Binary encounter

$$
p_{B 1}=p_{B 2}=p_{B 1 \mathrm{H}}=p_{B 2 \mathrm{H}}
$$

where $a$ denotes the accomodation coefficient for mass transfer, and $p_{A I}$ and $p_{A \mathbf{I}}$ are the saturated vapor pressures of substance $A$ at the surfaces of the inner and outer spheres, respectively. Using Eqs. (39) (41), Eqs. (36) $\sim(38)$ yield

$$
\begin{aligned}
p_{A \mathbf{I}}-p_{A \mathbf{I}}= & \left\{\frac{1}{a}+\frac{1-a}{a}\left(\frac{1}{\bar{R}^{*}}\right)^{2}\right\} \gamma \\
& +p_{B \mathbf{I}}\left[1-\exp \left\{-\zeta \gamma\left(1-\frac{1}{\bar{R}^{*}}\right)\right\}\right]
\end{aligned}
$$

On the other hand, the mass flux based on the outer surface of the inner sphere is given as follows;

$$
\left.\begin{array}{rl}
N_{A \mathrm{I}} & =\int m_{A} \xi_{A R} f_{A} d \boldsymbol{\xi}_{A}=m_{A} u_{A R \mathrm{I}} \\
& =\left(\frac{m_{A} k T}{2 \pi}\right)^{1 / 2}\left(n_{A 1}-n_{A 2}\right)=\left(\frac{m_{A}}{2 \pi k T}\right)^{1 / 2} \gamma \\
k_{g} & =\frac{N_{A \mathrm{I}}}{p_{A \mathrm{I}}-p_{A \mathrm{I}}}=\left(\frac{m_{A}}{2 \pi k T}\right)^{1 / 2} \frac{\gamma}{p_{A \mathrm{I}}-p_{A \mathbf{I}}} \\
M & =-2 p_{B 1 \mathrm{I}} \exp \left(\frac{\zeta \gamma}{\bar{R}^{*}}\right) \\
\delta & =2 p_{A \mathrm{I}}-\left(\frac{2}{a}-1\right) \gamma-M \exp (-\zeta \gamma)
\end{array}\right\}
$$

When $p_{A 11}, p_{A 21}$ and $p_{B 11}$ are known, one can obtain the value of $\gamma$ from Eq. (42) and then the mass transfer rate from Eq. (43) also.

It could be easily verified that Fq. (43) approaches to Hertz-Kundsen's equation under highly rarefied pressures and to Stefan's equation under ordinary pressures.

If $p_{B 1}$ I approaches to zero, Eq. (42) reduces to the following equation,

$$
r \cong \frac{1}{F(a)}\left(p_{A \mathrm{I}}-p_{A \mathbf{I}}\right)
$$

where

$$
F(a)=\frac{1}{a}+\frac{1-a}{a}\left(\frac{1}{\bar{R}^{*}}\right)^{2}
$$

Therefore

$$
N_{A \mathrm{I}} \cong \frac{1}{F(a)}\left(\frac{m_{A}}{2 \pi k T}\right)^{1 / 2}\left(p_{A \mathrm{I}}-p_{A \mathrm{I}}\right)
$$

This is Hertz-Knudsen's equation.

If $p_{B 1}$ takes so large value that the first term in the right hand side of Eq. (42) is negligibly smaller than the second, then one obtains

$$
\exp \left\{-\zeta \gamma\left(1-\frac{1}{\bar{R}^{*}}\right)\right\} \cong \frac{1}{p_{B 1 \mathbf{I}}}\left(p_{A \mathbf{I}}+p_{B 1 \mathbf{I}}-p_{A 1}\right)
$$




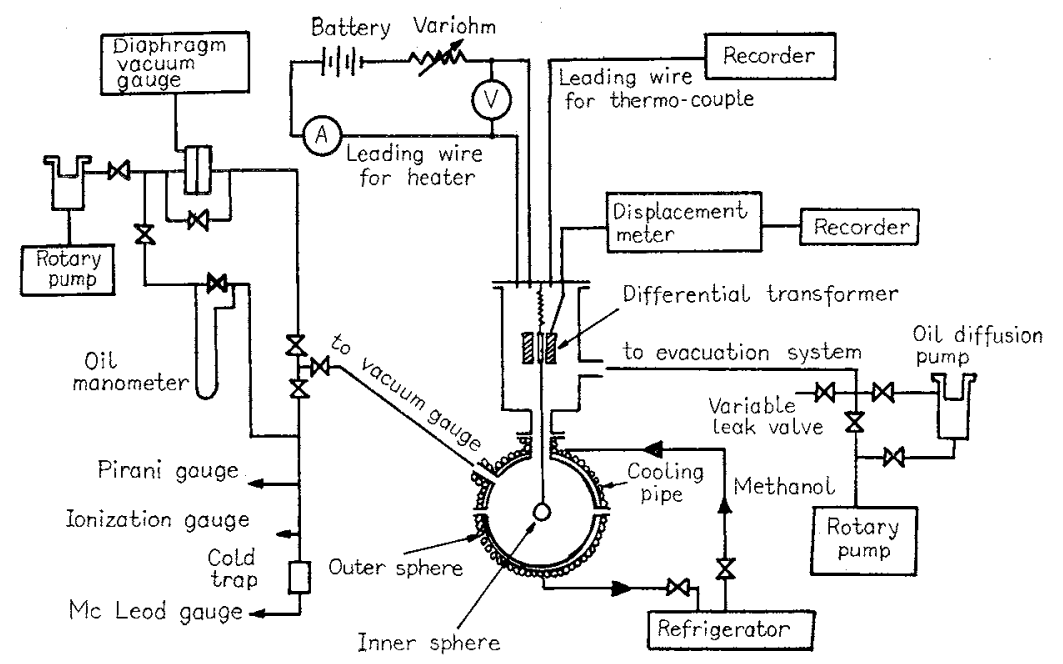

Fig. 3

Schematic diagram of apparatus

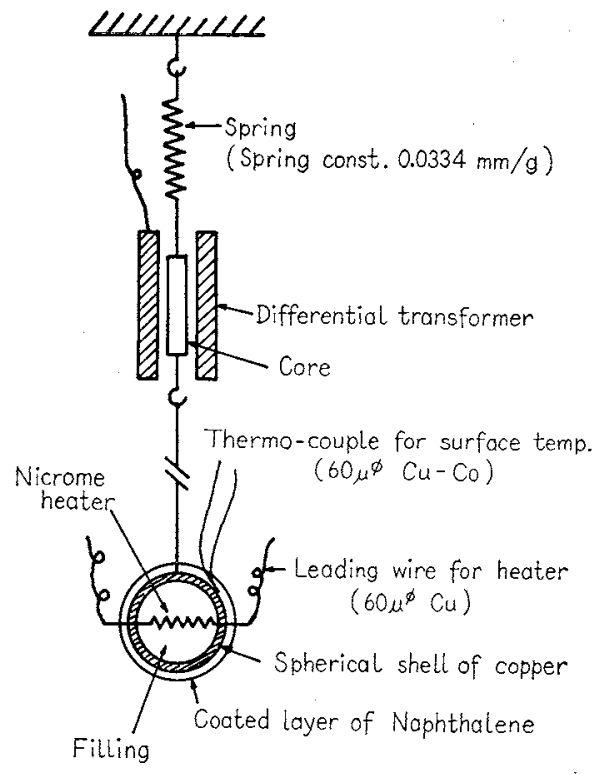

Fig. 4 Inner sphere and mechanism of weighing

$$
\begin{gathered}
p_{A \mathrm{II}}+p_{B 1 \mathrm{I}}-p_{A \mathrm{I}} \cong \mathrm{p}_{t}-p_{A \mathrm{I}} \cong p_{B 1 \mathrm{I}} \\
\gamma \cong-\left\{\zeta\left(1-\frac{1}{\bar{R}^{*}}\right)\right\}^{-1} \ln \left(\frac{p_{B 1 \mathrm{I}}}{p_{B 1 \mathrm{II}}}\right)
\end{gathered}
$$

because the value of $p_{t}$ is approximately constant.

Therefore

$$
\begin{aligned}
N_{A \mathrm{I}} & \cong-\left(\frac{m_{A}}{2 \pi k T}\right)^{1 / 2}\left(\frac{m_{A} \pi}{2 k T}\right)^{1 / 2} \frac{2 p_{t}\left[D_{A B}\right]}{R_{\mathrm{I}}\left(1-1 / \bar{R}^{*}\right)} \ln \left(\frac{p_{B 1 \mathrm{I}}}{p_{B 1 \mathrm{I}}}\right) \\
& \cong \frac{m_{A} p_{t}\left[D_{A B}\right]}{k T R_{\mathrm{I}}\left(1-1 / \bar{R}^{*}\right)} \frac{p_{A \mathrm{I}}-p_{A \mathrm{I}}}{\left(p_{B}\right)_{l m}}
\end{aligned}
$$

The above equation is Stefan's equation.

The distributions of the total pressure and the partial pressure of component $\mathrm{A}$ are expressed by

$$
\begin{aligned}
p_{t}= & \frac{1}{2}\left\{(1-\sin \alpha)\left(p_{A 1}+p_{B 2}\right)\right. \\
& \left.+(1+\sin \alpha)\left(p_{A 2}+p_{B 2}\right)\right\} \\
= & \frac{1}{2}(\delta-r \sin \alpha)
\end{aligned}
$$

and

$$
p_{A}=\frac{1}{2}\left\{\delta+M \exp \left(-\frac{\zeta \gamma}{\bar{R}}\right)-\gamma \sin \alpha\right\}
$$

\section{Experimental Apparatus and Procedures}

The experimental apparatus used is schematically shown in Fig. 3.

The measurements of the sublimation rate of naphthalene coated on the inner sphere were performed under reduced pressure. The inner sphere was placed at the center of the outer sphere working as a condensating surface.

The outer sphere, the sublimation chamber $\left(150^{\operatorname{mm} \phi} \mathrm{I}\right.$. D. $\times 160^{\mathrm{mm} \phi}$ O.D.), was made of bronze and jacketed by the copper cooling pipe through which methanol cooled by a refrigerator, $0 \sim-40^{\circ} \mathrm{C}$, was circulated.

The inner sphere (about $22 \sim 30^{\mathrm{mm} \phi}$ ) was the copper spherical shell having $1.5 \mathrm{~mm}$ thickness in which the electric heater was provided, and was coated with naphtalene about to $2 \mathrm{~mm}$ thickness and located concentrically in the outer sphere (see Fig. 3 and Fig. 4). The coating of naphthalene was carried out by dipping the inner sphere into a bath of molten naphthalene purified by recrystallization. The inner sphere was electrically heated by supplying D.C. current and the surface temperature of naphthalene was regulated to be constant by a variohm and determined from the temparature of the surface of inner sphere taking account of the difference through the layer.

To determine the rate of sublimation, the weight loss of naphthalene on the inner sphere was measured by the displacement meter using the differential transformer (see Fig. 3 and Fig. 4) and recorded continuously.

For pressure measurement, Atlas-MMM diaphragm vacuum gauge for relatively low pressures and oil manometer for relatively high pressures were used. The measured value of the pressure at the outer sphere by using vacuum gauge, $p_{D}$, is

$$
\begin{aligned}
p_{D} & =\frac{1}{2}\left\{\left(1+\cos 2 \alpha_{\mathbb{I}}\right) p_{A 1}+\left(1-\cos 2 \alpha_{\mathbb{I}}\right) p_{A 2 \mathbb{I}}\right\}+p_{B 1 \mathbb{I}} \\
& =\left(\frac{1}{\bar{R}^{*}}\right)^{2} p_{A 1 \mathbb{I}}+\left\{1-\left(\frac{1}{\bar{R}^{*}}\right)^{2}\right\} p_{A 2 \mathbb{I}}+p_{B 1 \mathbb{I}}
\end{aligned}
$$

From Eq. (55), the value of $p_{B 11}$ can be calculated.

In the experiments, the total pressures of the apparatus were kept constant in the range $10^{-1} \sim 10^{-4} \mathrm{mmHg}$ by a variable leak valve. 


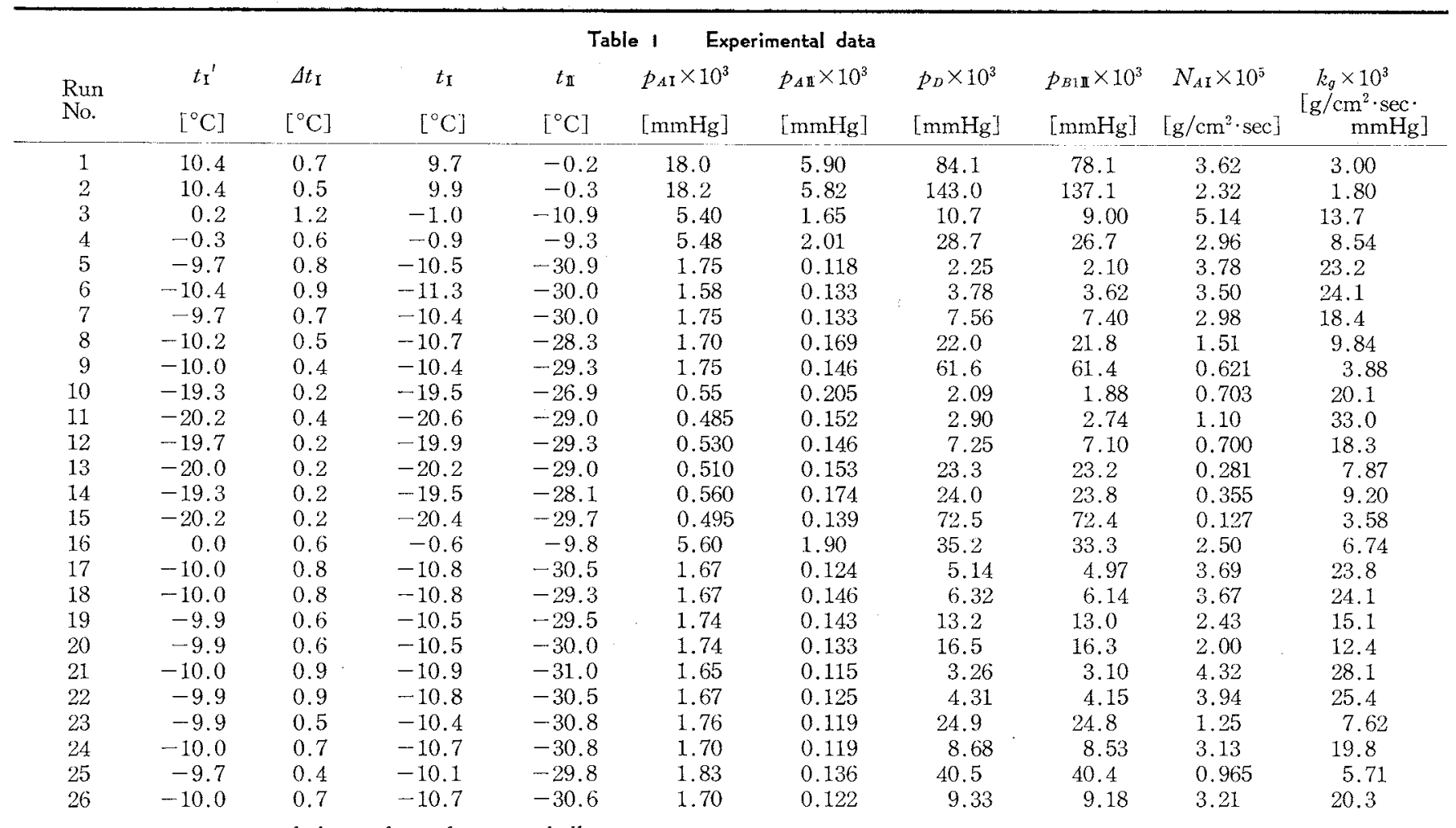

$t_{1}$ : temperature of the surface of copper shell

$\Delta t_{1}$ : temperature difference through the coated layer of naphthalene

The data of vapor pressure, thermal conductivity and diffusivity of naphthalene were cited from I.C. T..

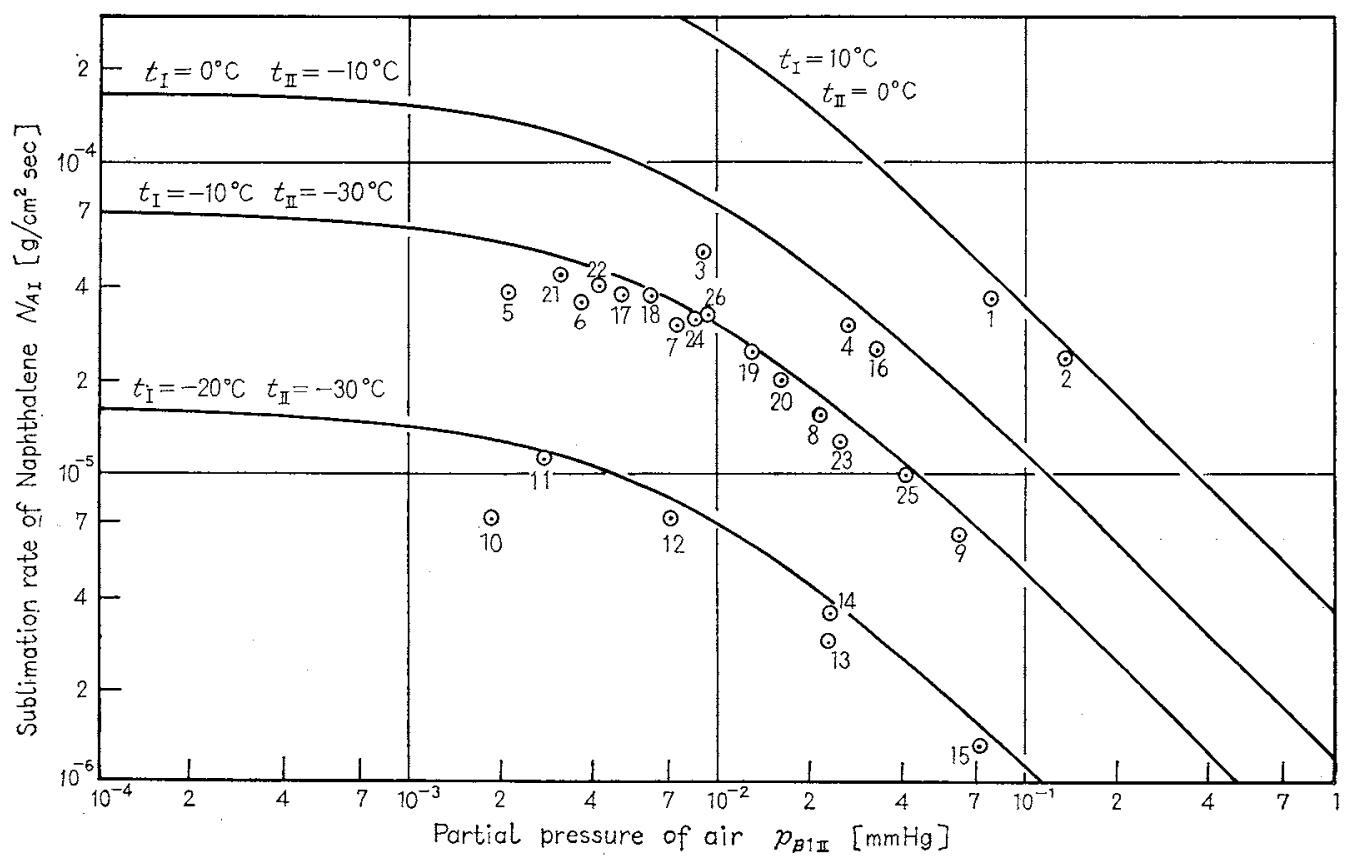

Fig. 5 Comparison between analytical and experimental results

The procedures of the experiments were as follows; after hanging the coated inner sphere from a hook below the core of differential transformer, the sublimation chamber was evacuated for several minutes to the prescribed pressure and the outer sphere was cooled to the prescribed temperature.

Then the inner sphere was electrically heated for the surface temperature to be constant. The weight loss of the inner sphere and the temperatures of the surfaces were recorded continuously.

The measurements and regulations of the temperatures of the inner and outer sphere were carried out within $\pm 0.1^{\circ} \mathrm{C}$ and $\pm 0.4^{\circ} \mathrm{C}$ in errors, respectively.

The relative error on the determination of sublimation rate was estimated to be in $\pm 2 \%$. 


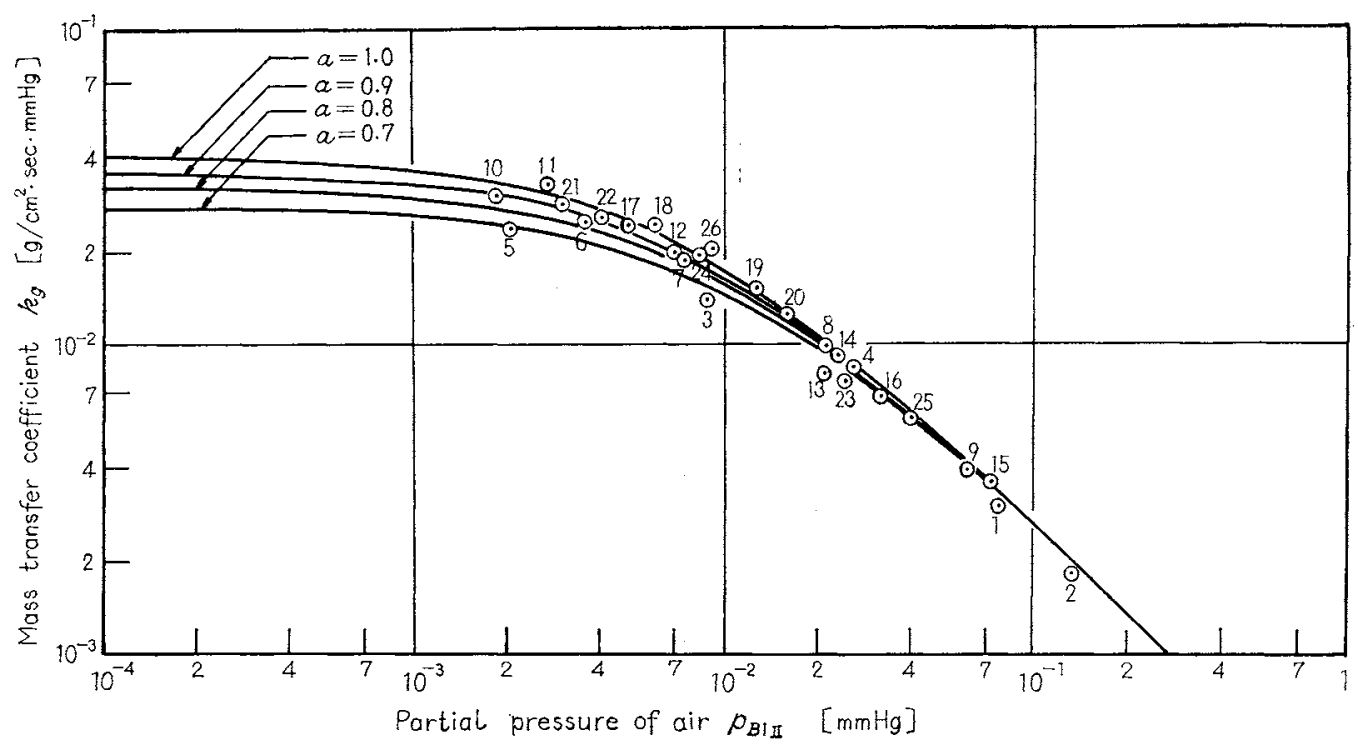

Fig. 6 Comparison detween anaiyticai and experimental results

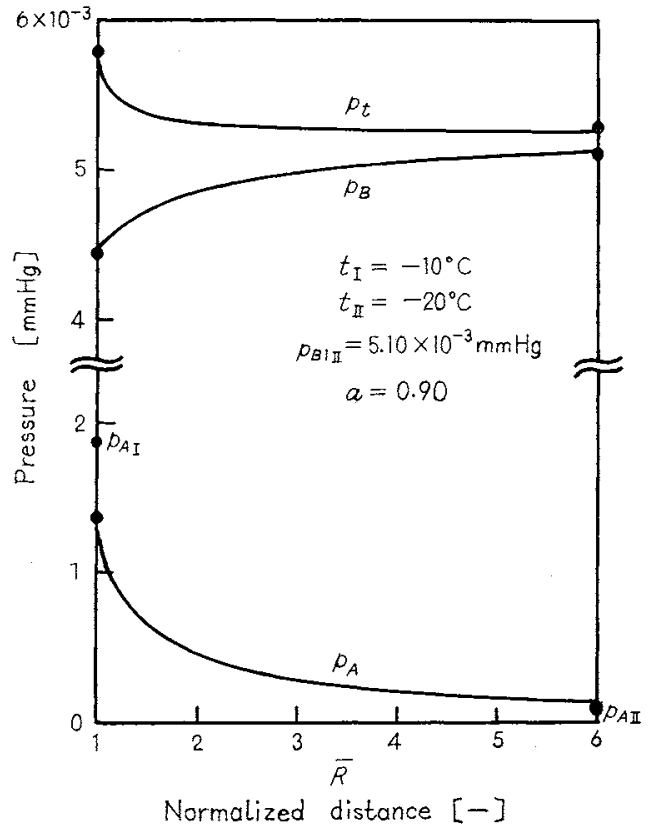

Fig. 7 Distributions of pressure

\section{Experimental Results and Discussions}

The mass transfer rate based on the surface of the inner sphere, $N_{A I}$, was calculated from the rate of weight loss of naphthalene by using the following equation,

$$
N_{A \mathbf{I}}=-\frac{1}{4 \pi R_{\mathrm{I}}{ }^{2}} \frac{d W}{d t}
$$

The value of $p_{B 1}$ was calculated from the value of $p_{D}$ measured by the vacuum gauge by using Eqs. (55), and (37).

$$
p_{D}=\gamma+p_{B 1 \mathbf{r}}+\frac{1}{2}\left\{-\gamma+\delta+M \exp \left(-\frac{\zeta \gamma}{\bar{R}^{*}}\right)\right\}
$$

From Eqs. (45) and (57), we obtain the following equation;

$$
p_{B 1 \mathrm{X}}=p_{D}-p_{A \mathrm{I}}-\frac{1}{a}\left(-\frac{1}{\bar{R}^{*}}\right)^{2} \gamma
$$

The experimental results are shown in Table 1. Further the values of $p_{B 1} \mathbf{m}$ in Table 1 were calculated from Eq. (58) assuming that the value of $a$ was equal to unity, because the error produced by this approximation was negligibly small in the final value.

In Fig. 5, the observed values of the sublimation rate are compared with the calculated values from Eq. (43). The observed values fairly agree with the calculated ones from the continuum to the transition region. It can be observed that in the free molecular region the observed values are a little smaller than the calculated ones as shown in Fig. 5.

The observed values of $k_{g}$ are also compared with the calculated values from Eq. (44) in Fig. 6. From Fig. 6 it may be said that the accomodation coefficient $a$ for naphthalene is equal about to 0.9 . It has been reported by Sherwood et $\mathrm{al}^{9{ }^{9}}$ that this coefficient was equal to 0.88 about at $-60^{\circ} \mathrm{C}$.

The distributions of the total pressure and the partial pressures of component $A$ and $B$ are calculated by using Eqs. (53) and (54). An example is shown in Fig. 7. The pressure-jump is observed distinctly on the surface of inner sphere and the total pressure changes steeply near the inner sphere.

\section{Conclusion}

The equation of mass transfer between concentric spheres under reduced pressures, especially under the transition regime, was derived through the gas kinetic theory using the two-sided Maxwellian distribution function. The observed values of the sublimation rate of naphthalene were compared with the values calculated from the equation derived in the present work and both were in good agreement.

\section{Acknowledgements}

We wish to thank Mr. Yoshihiro Konaka and Mr. Masashi Asaeda for technical assistance. This work was supported in part 
by the Kawakami Memorial Foundation, to which our thanks are due.

\section{Nomenclature}

$A_{1} \quad=$ constant defined by Eq. (25)

$a \quad=$ accomodation coefficient for mass transfer

$b \quad=$ impact parameter, or perpendicular distance from molecule $A$ to initial trajectory of molecule $B$ (see Fig. 2)

$\left[D_{A B}\right]=$ diffusion coefficient of molecule $A$ through molecule $B$

$F \quad=$ intermolecular force for the Maxwell molecule

$F(a)=$ the function of accomodation coefficient:

$$
\frac{1}{a}+\frac{1-a}{a}\left(\frac{1}{\bar{R}^{*}}\right)^{2}
$$

$f \quad=$ velocity distribution function of molecule

$\widetilde{K}_{A B}=$ constant in expression for central force field,

$$
F=\frac{\widetilde{K}_{A B}}{r^{5}}
$$

$k=$ Boltzmann constant

$\left[\mathrm{mmHg} \cdot \mathrm{cm}^{3} /{ }^{\circ} \mathrm{K}\right]$

$k_{g} \quad=$ mass transfer coefficient $\left[\mathrm{g} / \mathrm{cm}^{2} \cdot \mathrm{sec} \cdot \mathrm{mmHg}\right]$

$M \quad=$ integral constant defined in Eqs. $(36) \sim(38) \quad[\mathrm{mmHg}]$

$m \quad=$ mass of a molecule

[g]

$N_{A I}=$ mass transfer rate based on the inner sphere

$=$ number density of molecule

$=$ component of perssure tensor

$\left[\mathrm{g} / \mathrm{cm}^{2} \cdot \mathrm{sec}\right]$ $\left[1 / \mathrm{cm}^{3}\right]$ $[\mathrm{mmHg}]$

$=$ pressure defined by $p=n k T$

$[\mathrm{mmHg}]$

$Q \quad=$ physical quantity

$\Delta Q=$ change in $Q$ produced by collision; collision integral

$R=$ radial distance from the center of the inner sphere

$\bar{R} \quad=$ normalized distance from the center of the inner sphere; $\bar{R}=R / R_{\mathrm{I}}$

$\bar{R}^{*} \quad=R_{\mathbf{H}} / R_{\mathbf{I}}$

$r \quad=$ distance between two molecules colliding each other

$T=$ absolute temperature $\quad[\mathrm{cm}]$

$t=$ time [sec]

$V \quad=$ relative velocity between two interacting molecules

$W \quad=$ weight of the inner sphere

\section{Greek letters}

$\alpha \quad=$ angle defined by Eq. (4)

$\beta=$ variable concerned in variable $b$ (cf. literature 5 ) $\gamma \quad=$ integral constant defined by Eq. (31)

$\gamma^{\prime}=$ integral constant defined by Eq. (14)

$=$ integral constant defined by Eq. (21)

$=$ angle between plane of the orbit and plane containing the original relative velocity and the $X$-axis in a binary collision (see Fig. 2)

- scattering angle, or angle between relative velocity before and after collision

$=$ velocity of molecule

$=$ velocity vector of molecule

$=$ angle defined in Fig. 1

$=$ constant defined by Eq. (35)

Suffix

$A=$ diffusing component

$B \quad=$ stagnant component

$R=$ radial direction on polar co-ordinate

$D=$ pressure directed outwardly at the inner surface of the outer sphere

$=$ total pressure

$=\theta$-direction on polar co-ordinate

$=\phi$-direction on polar co-ordinate

$=$ inside of the wedge of "influence" (see Fig. 1)

$=$ outside of the wedge of "influence" (see Fig. 1)

$=$ the inner sphere

$=$ the outer sphere

$>=$ averaged value

$=$ after collision

\section{Literature cited}

1) Chapman, S. and T. G. Cowling: "The Mathematical Theory of Non-Uniform Gases" (Cambridge, 1964)

2) Lees, L. and C. Liu: Phys. Fluid. 5, 1137 (1962)

3) Madden, A. J. : A. I. Ch. E. J., 5, 135 (1959)

4) Madden, A. J. and F. J. Halfen: A. I. Ch. E. J., 7, 160 (1961)

5) Maxwell, J. C.: Phil. Trans. Roy. Soc. 157, 49 (1867)

6) Mikami, H., Y. Endo and Y. Takashima: Int. J. Heat Mass Transfer, 9, 1435 (1966)

7) Mott-Smith, H. M. : Phys. Review, 82, 885 (1951)

8) Sherwood, T.K. and N.E. Cooke: A. I. Ch. E. J., 3, 37 (1957)

9) Sherwood, T.K. and C. Johannes: A.I.Ch. E. J., 8, 590 (1962)

10) Smoluchowski. M.: Ann. Phys., 35, 984 (1911)

11) Uyeha, H., T. Kajiura and O. Yoshikawa: Chem. Eng. (Japan), 24, $274(1960)$ 